\title{
TNF can activate RIPK3 and cause programmed necrosis in the absence of RIPK1
}

\author{
DM Moujalled ${ }^{1,2}$, WD Cook ${ }^{3}$, T Okamoto $^{4}, \mathrm{~J} \mathrm{Murphy}^{1,2}$, KE Lawlor $^{1,2}, \mathrm{JE}$ Vince $^{1,2}$ and DL Vaux ${ }^{\star, 1,2}$
}

Ligation of tumor necrosis factor receptor 1 (TNFR1) can cause cell death by caspase 8 or receptor-interacting protein kinase 1 (RIPK1)- and RIPK3-dependent mechanisms. It has been assumed that because RIPK1 bears a death domain (DD), but RIPK3 does not, RIPK1 is necessary for recruitment of RIPK3 into signaling and death-inducing complexes. To test this assumption, we expressed elevated levels of RIPK3 in murine embryonic fibroblasts (MEFs) from wild-type (WT) and gene-deleted mice, and exposed them to TNF. Neither treatment with TNF nor overexpression of RIPK3 alone caused MEFs to die, but when levels of RIPK3 were increased, addition of TNF killed WT, Ripk $1^{-I-}$, caspase $8^{-I-}$, and $\mathrm{Bax}^{-I-} / \mathrm{Bak}^{-I-} \mathrm{MEFs}$, even in the presence of the broad-spectrum caspase inhibitor Q-VD-OPh. In contrast, Tnfr1 ${ }^{-1-}$ and Tradd $^{-l-}$ MEFs did not die. These results show for the first time that in the absence of RIPK1, TNF can activate RIPK3 to induce cell death both by a caspase 8-dependent mechanism and by a separate Bax/Bak- and caspase-independent mechanism. RIPK1 is therefore not essential for TNF to activate RIPK3 to induce necroptosis nor for the formation of a functional ripoptosome/necrosome.

Cell Death and Disease (2013) 4, 465; doi:10.1038/cddis.2012.201; published online 17 January 2013

Subject Category: Experimental Medicine

By binding to tumor necrosis factor receptor 1 (TNFR1), TNF can activate both transcription factors, such as AP-1 and $\mathrm{NF}-\kappa \mathrm{B}$, as well as cell death mechanisms. ${ }^{1}$ Because most cell lines are not killed by the addition of TNF alone, but many die when TNF is added together with inhibitors of transcription or translation, such as actinomycin D or cycloheximide $(\mathrm{CHX}),{ }^{2}$ these transcription factor pathways appear to promote cell survival. Consistent with this notion, cell lines mutant for the p65/RelA component of NF- $k$ B are killed by TNF alone. ${ }^{3}$ These observations show that upon ligation, TNFR1 not only activates the NF- $\kappa$ B pathway but also triggers cell death pathways that can be blocked by an NF- $\kappa$ B-dependent process.

TNF activates a number of different death mechanisms depending of the cell type and circumstances. In many cell types, including mouse embryonic fibroblasts (MEFs), cell death triggered by TNF (plus CHX), or by related ligands such as TRAIL or FasL, requires the presence of the adaptor protein Fas-associated death domain (FADD) and the protease caspase $8 .^{4,5}$ The receptors for these ligands bear cytoplasmic death domains (DDs) that allow them to bind directly or indirectly to FADD, which in turn binds to the death effector domains of procaspase 8 , causing it to activate. $^{6}$

Although cell death triggered by ligation of death receptors can often be blocked by the caspase 8 inhibitor $\mathrm{crmA}^{7,8}$ or by pancaspase inhibitory compounds such as Q-VD-OPh ${ }^{9}$ or
z-VAD-FMK, ${ }^{10}$ not all cells are protected. For example, murine fibrosarcoma L929 cells were killed more efficiently by TNF in the presence of Z-VAD-FMK, ${ }^{11,12}$ and Z-VAD-FMK did not prevent the death of U937 cells treated with TNF, or HT29 cells treated with TNF plus an inhibitor of apoptosis protein (IAP) antagonist (smac-mimetic). ${ }^{13}$ Instead, these cells died displaying a characteristic appearance termed 'necroptosis'.'11

Necroptosis, or programmed necrosis, refers to a caspase 8-independent death mechanism triggered by the receptorinteracting protein kinase 1 and 3 (RIPK1 and RIPK3). ${ }^{11,14-18}$ Necroptosis is thought to be dependent on the enzymatic activity of RIPK1, as suggested by the protection conveyed by the RIPK1 kinase inhibitor necrostatin. ${ }^{19}$ Because RIPK1, but not RIPK3, bears a DD that can interact with the TNFR1, ${ }^{20}$ it has been suggested that upon binding of TNF, RIPK1 is recruited to TNFR1 either directly via its DD or indirectly by a DD-bearing adaptor, such as TNF receptor-associated death domain (TRADD), to form a complex on the cytoplasmic domain of TNFR1 ${ }^{21}$ In cells destined to die, this complex is released from TNFR1, and recruits other proteins, such as FADD and caspase 8 to induce apoptosis, or interacts with RIPK3 to cause necroptosis. ${ }^{22,23}$ RIPK1 is thought to have a crucial role in recruiting RIPK3 by binding via their shared RIP homotypic interaction motifs (RHIMs). ${ }^{22,24,25}$ Therefore, according to this model, RIPK1 recruitment of RIPK3 is essential for ligated TNFR1 to signal necroptosis. ${ }^{26,27}$

\footnotetext{
${ }^{1}$ The Walter and Eliza Hall Institute of Medical Research, 1 G Royal Parade, Melbourne, Victoria, Australia; ${ }^{2}$ Department of Medical Biology, The University of Melbourne, Parkville, Victoria, Australia; ${ }^{3}$ Department of Biochemistry, La Trobe Institute for Molecular Science, La Trobe University, Kingsbury Drive Bundoora, Melbourne, Victoria, Australia and ${ }^{4}$ Department of Molecular Virology, Research Institute for Microbial Diseases, Osaka University, Yamadaoka, Suita, Osaka, Japan

${ }^{*}$ Corresponding author: DL Vaux, Department of Biochemistry, La Trobe Institute for Molecular Science, La Trobe University, Kingsbury Drive Bundoora, Melbourne, Victoria 3086, Australia. Tel: +61 39345 4129; E-mail: vaux@ wehi.edu.au

Keywords: TNF; necroptosis; receptor-interacting protein kinases; smac-mimetic; inhibitor of apoptosis proteins TNF

Abbreviations: CHX, cycloheximide; DD, death domain; TNF, tumor necrosis factor; TNFR1, TNF receptor 1; RIPK1/3, receptor-interacting protein kinases; IAPs, inhibitor of apoptosis proteins; TRADD, TNF receptor-associated death domain; FADD, Fas-associated death domain

Received 28.11.12; accepted 4.12.12; Edited by G Melino
} 
To determine the roles and requirements for TRADD, FADD, caspase 8, RIPK1, RIPK3, and Bax/Bak for TNFinduced cell death, we derived MEFs from gene-deleted mice, and treated them with TNF, in the presence or absence of exogenously expressed RIPK3. We found that addition of TNF activated both caspase 8- and RIPK3-dependent death pathways, even in the absence of RIPK1. Our results show that although TNFR1 and TRADD were necessary for TNF to activate RIPK3 to cause cell death, RIPK1 and FADD were not.

\section{Results}

Elevation of RIPK3 allows TNF to cause death of MEFs. To determine whether overexpression of RIPK3 alone was sufficient to cause death of MEFs, we infected wild-type (WT) MEFs with a 4-hydroxytamoxifen (4HT)inducible lentiviral vector expressing FLAG-tagged RIPK3 (Figure 1a). As expected, in the absence of 4HT, cells treated with TNF for $24 \mathrm{~h}$ did not die, whereas those treated with TNF plus a smac-mimetic compound, which depletes cells of clAP $1,{ }^{7}$ did die (Figure $1 \mathrm{~b}$, upper panels). Induction of FLAG-RIPK3 by $4 \mathrm{HT}$ also failed to kill the cells, but when TNF was added to cells in which FLAG-RIPK3 had been induced, they died (Figure 1b, lower panels). These results show that although elevation of RIPK3 levels alone is not sufficient to cause cell death, it can sensitize MEFs to killing by TNF, even when clAP1 is present.

TNFR1 and TRADD are required for TNF to cause death of MEFs with elevated RIPK3. TNFR1 signaling involves formation of two distinct signaling complexes, the transient membrane-associated TNFR1 signaling complex (complex I) and the cytoplasmic signaling complex termed complex II. ${ }^{21}$ To determine components required for TNF to cause death of cells expressing elevated RIPK3, we tested MEF lines that were mutant for proteins implicated in TNF signaling. We infected Tnfr1 $^{-1-}$, Tnfr2 ${ }^{-1-}$, and Tradd $^{-1-}$ MEFs with the inducible lentiviral vector expressing FLAG-tagged RIPK3 (Supplementary Figure 1a).

Unlike WT MEFs and those mutant for TNFR2, the Tnfr1 $^{-1-}$ and Tradd $^{-1-}$ MEFs were not killed when RIPK3 expression was induced with $4 \mathrm{HT}$ and TNF was added (Figure 2). Therefore, both TNFR1 and TRADD are required for TNF to activate RIPK3's killing function.

TNF can activate RIPK3 and cause cell death in the absence of caspase 8 and RIPK1. Numerous reports have described 'programmed necrosis' or 'necroptosis' as forms of cell death mediated by the serine/threonine kinases RIPK1 and RIPK3 that are independent of caspase $8 .^{11,14,18}$ To determine whether TNF-triggered death of cells with elevated RIPK3 required caspase 8 , we generated caspase $8^{-1-}$ MEFs by deleting loxP-flanked caspase 8 alleles in vitro (Supplementary Figure 1b), and then infected them with the 4HT-inducible RIPK3 lentivirus.

First, we compared the sensitivity of WT, caspase $8^{-1-}$, and Ripk $1^{-1-}$ MEFs to killing by TNF plus smac-mimetic (Figure 3a). Unlike WT MEFs, which were efficiently killed by TNF plus smac-mimetic, very few of the Ripk $1^{-1-}$ and caspase $8^{-1-}$ cells died, indicating that when IAPs are depleted by smac-mimetic, TNF triggers death of MEFs by a RIPK1- and caspase 8-dependent mechanism. ${ }^{28,29}$ This requirement of RIPK1 and caspase 8 for killing of MEFs by TNF plus smac-mimetic is similar to that observed in neuroblastoma cells treated with TRAIL and IAP inhibitor. ${ }^{30}$

To determine whether TNF-triggered death of MEFs with elevated RIPK3 used the same mechanism as when TNF caused death of IAP-depleted cells, we infected the caspase $8^{-1-}$ and Ripk $1^{-1-}$ MEFs with the inducible

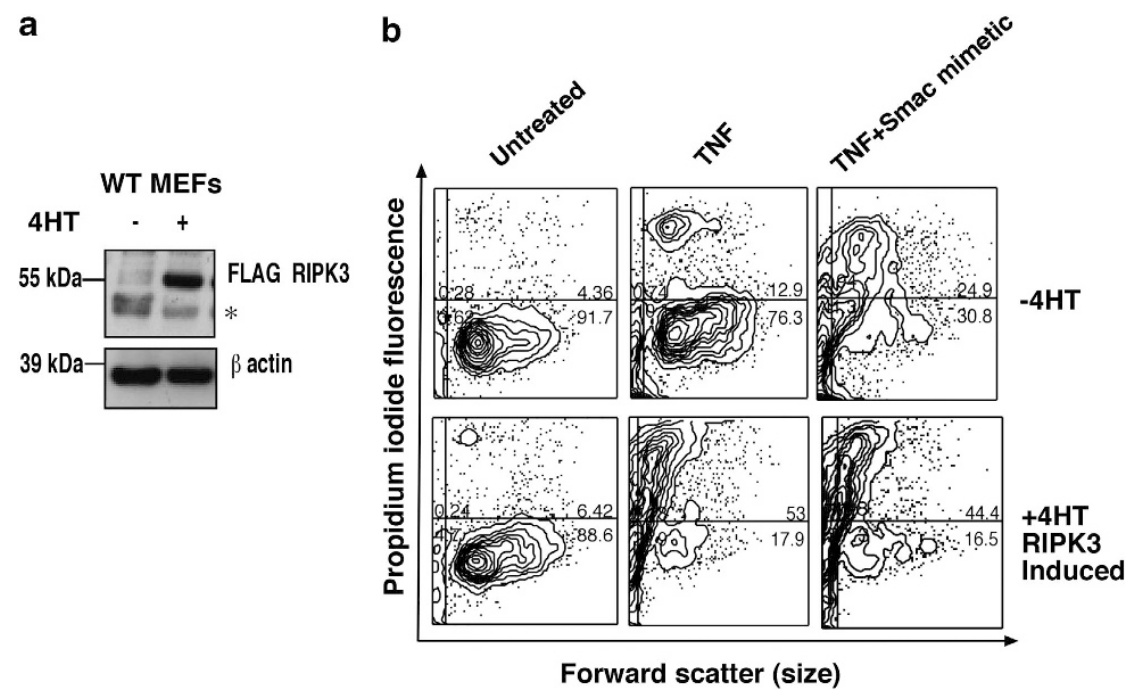

Figure 1 TNF causes death of cells with elevated RIPK3. (a) WT MEFs were infected with a lentiviral vector-expressing FLAG RIPK3. Cells were induced with 10 nM 4HT for $24 \mathrm{~h}$, and lysates were probed for FLAG. $\beta$-Actin was used as a loading control. *, indicates a nonspecific band. (b) Expression of FLAG-RIPK3 in MEFs was induced with $10 \mathrm{nM} 4 \mathrm{HT}$ for $24 \mathrm{~h}$ (lower panels), and cells were then treated with $100 \mathrm{ng} / \mathrm{ml}$ TNF or TNF plus $500 \mathrm{nM}$ smac-mimetic for a further $24 \mathrm{~h}$. Cells were stained with PI and analyzed by flow cytometry to detect loss of plasma membrane integrity 


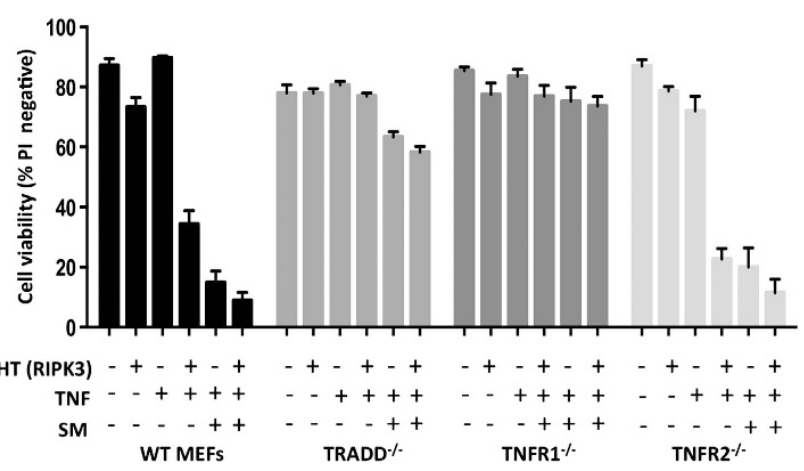

Figure 2 TNFR1 and TRADD are necessary for TNF to cause death of MEFs treated with smac-mimetic or with elevated RIPK3. FLAG-RIPK3 was induced in WT, Tnfr1 ${ }^{-1-}$, Tnfr2 $^{-1-}$, and Tradd ${ }^{-1-}$ MEFs with $10 \mathrm{nM} 4 \mathrm{HT}$ for $24 \mathrm{~h}$. Cells were then treated with $100 \mathrm{ng} / \mathrm{ml}$ TNF or TNF plus $500 \mathrm{nM}$ smac-mimetic for a further $24 \mathrm{~h}$, stained with PI, and analyzed by flow cytometry. Columns show mean \pm S.E.M., where $n=3$ independently performed experiments

lentiviral vector bearing FLAG-tagged RIPK3 (Figure $3 \mathrm{~b}$ and Supplementary Figure 1c). When FLAG-RIPK3 was induced in Ripk $1^{-1-}$ and caspase $8^{-1-}$ MEFs, little cell death occurred (Figure 3c). There was also little cell death when TNF was added alone. However, addition of TNF to cells in which RIPK3 had been induced by $4 \mathrm{HT}$ strongly induced cell death, both in short-term (Figure 3c) and long-term clonogenic survival assays (Figure $3 \mathrm{~d}$ ). Therefore, neither RIPK1 nor caspase 8 is required for TNF to induce cell death when RIPK3 levels are elevated, but both are needed for TNF to kill cells in which IAPs are depleted by a smac-mimetic (Figure 3b). This indicates that while death of MEFs caused by TNF plus smac-mimetic requires both RIPK1 and caspase 8 activity, when RIPK3 levels are elevated, TNF can induce a RIPK1- and caspase 8-independent death mechanism.

RIPK3 killing triggered by TNF does not require Bax/Bak. Although several groups have shown that RIPK1 and RIPK3 can trigger a caspase-independent, necrotic form of cell death, a number of different effector mechanisms have been proposed. For example, it has been reported that in some cells, RIP kinases cause cell death by stimulating production of reactive oxygen species (ROS) or by disrupting mitochondrial integrity. ${ }^{14}$ In addition, Zhang et al. ${ }^{18}$ suggested that RIPK3 generates ROS by interacting with the mitochondrial metabolic enzymes glutamate dehydrogenase and glycogen phosphorylase, whereas another group proposed that RIPK3 induces cell death by interacting with MLKL and PGAM5, and activating DRP-1 to cause mitochondrial dysfunction; ${ }^{17,22}$ a fourth group found that binding to DNA-dependent activator of interferon regulatory factors (DAI) was necessary for RIPK3 to cause necrosis; ${ }^{31}$ and a fifth group reported that Bax or Bak was necessary for TNF-induced necrosis. ${ }^{32}$ To determine whether RIPK3 causes cell death by activating the intrinsic (Bax/Bak-dependent) pathway, we infected $\mathrm{Bax}^{-1-} / \mathrm{Bak}^{-1-} \mathrm{MEFs}$ with an inducible lentiviral vector encoding FLAG RIPK3 (Supplementary Figure 1d), and treated the cells with TNF. As shown in Figure 4, when RIPK3 levels were elevated, TNF caused a similar amount of death of $\mathrm{Bax}^{-1-} / \mathrm{Bak}^{-1-}$ as of WT
MEFs, indicating that activated RIPK3 can kill cells by a Bax/ Bak-independent mechanism. Furthermore, as the broadspectrum caspase inhibitor Q-VD-OPh was not able to prevent death of the $\mathrm{Bax}^{-1-} / \mathrm{Bak}^{-1-}$ MEFs, RIPK3 must be able to activate a death mechanism that is not only independent of Bax and Bak but also does not require caspase activity.

When RIPK3 levels are elevated, TNF activates caspases whether RIPK1 is present or not. Even though it was clear that when RIPK3 levels were elevated, TNF could trigger cell death by a caspase 8-independent mechanism (Figures $3 \mathrm{c}$ and d), TNF is also capable of activating caspase 8 , because when IAPs were depleted by smac-mimetic, TNF killed WT, but not caspase $8^{-1-}$ cells (Figures $3 \mathrm{c}$ and d). To see whether TNF could activate caspase 8 when RIPK3 levels were elevated, we treated WT and Ripk $1^{-1-}$ MEFs with $4 \mathrm{HT}$ to induce FLAG-RIPK3, added TNF, and after $4 \mathrm{~h}$ lysed the cells and analyzed them by western blot (Figure $5 \mathrm{a}$ ). Addition of TNF was able to trigger processing of caspase 8 , but only when FLAG-RIPK3 was induced. Strikingly, caspase 8 processing was triggered by the addition of TNF both in the WT and Ripk $1^{-1-}$ MEFs, indicating that when RIPK3 levels are elevated, TNFR1 can signal via RIPK3 to activate caspase 8 even in the absence of RIPK1.

To further analyze the caspase pathways that were activated by TNF in cells with elevated RIPK3, we performed additional experiments in Ripk $1^{-1-}$ and caspase $8^{-1-}$ MEFs, using western blots to test for processing of caspase 3 and cleavage of PARP, and taking cells from duplicate wells to assess their viability by flow cytometry.

As shown in Figure 5b, TNF triggered the processing and activation of caspase 3 and PARP cleavage only in cells with elevated RIPK3. Furthermore, when RIPK3 was elevated, TNF caused activation of caspase 3 in Ripk $1^{-1-}$ MEFs, but not in caspase $8^{-1-}$ MEFs.

Taken together, these experiments show that when ligated, TNFR1 can signal to RIPK3 independently of RIPK1, and if its levels are high enough, RIPK3 can activate caspase 8 and caspase 3 even if RIPK1 is absent. Nevertheless, activated caspase 8 was not required for most of the death when TNF was added to cells with elevated RIPK3, because Q-VD-OPh was not able to prevent their death (Figure 5c, WT and Ripk $1^{-1-}$ gray columns), and cell death still occurred even in the caspase $8^{-1-}$ MEFs (Figure 5c, light gray columns).

TNF can activate RIPK3 in the absence of FADD. To determine whether FADD was required for TNFR1 to activate RIPK3, we transfected the RIPK3-inducible construct into Fadd ${ }^{-1-}$ MEFs. When these cells were treated with 4HT (to induce RIPK3) and TNF, they did not die. However, as western blots showed that the Fadd ${ }^{-1}$ MEFs expressed relatively low levels of MLKL, a protein thought to be necessary for RIPK3-induced cell death, ${ }^{22,33}$ we hypothesized that the Fadd ${ }^{-1}$ MEFs might be surviving because of low levels of MLKL, rather than because they lacked FADD. To resolve this, we transfected the $\mathrm{Fadd}^{-1-}$ cells with a second vector bearing a doxycycline-inducible MLKL construct (Figure 6a). Addition of TNF to Fadd ${ }^{-1-}$ cells 

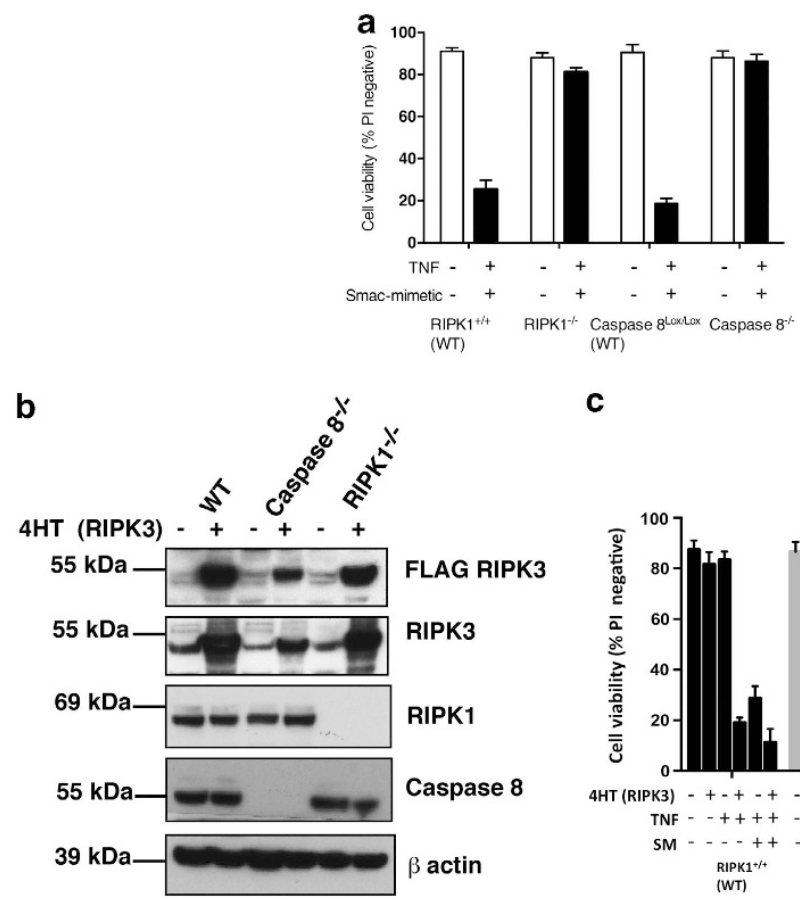

C

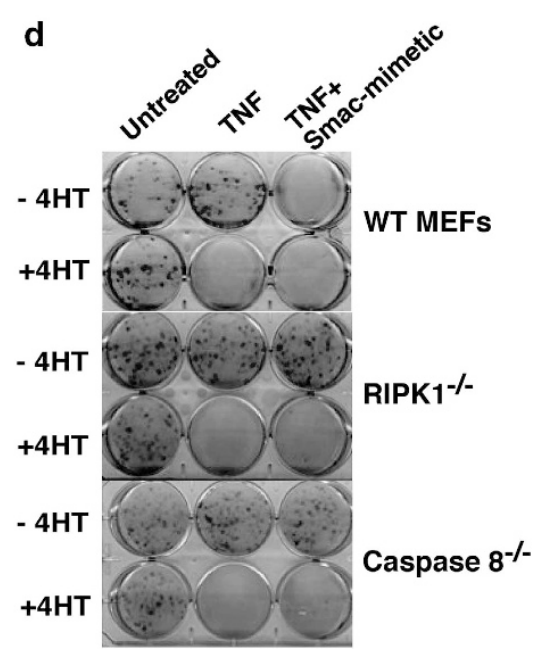

Figure 3 Both caspase 8 and RIPK1 are required for TNF plus smac-mimetic to cause cell death, but when levels of RIPK3 are elevated, TNF alone can induce cell death in the absence of caspase 8 or RIPK1. (a) MEF lines were untreated (white bars) or treated with $100 \mathrm{ng} / \mathrm{ml}$ TNF plus $500 \mathrm{nM}$ smac-mimetic (black bars) for $24 \mathrm{~h}$. Cell viability was determined by PI exclusion using a flow cytometer. Columns show mean \pm S.E.M., where $n=3$ independently performed experiments. (b) WT, caspase $8^{-1-}$, and Ripk $1^{-1-}$ MEFs bearing the inducible FLAG RIPK3 vector were untreated or treated with $10 \mathrm{nM} 4 \mathrm{HT}$ for $24 \mathrm{~h}$, and lysates were probed with antibodies to reveal levels of endogenous or induced FLAG-tagged proteins. (c) Cell lines were induced with $10 \mathrm{nM} 4 \mathrm{HT}$ for $24 \mathrm{~h}$, and then treated, where indicated, with $100 \mathrm{ng} / \mathrm{ml}$ TNF and $500 \mathrm{nM}$ smacmimetic (SM) for a further $24 \mathrm{~h}$, and viability determined by flow cytometric analysis of PI exclusion. Columns show mean \pm S.E.M., where $n=3$ independently performed experiments. (d) Cell lines were induced with $10 \mathrm{nM} 4 \mathrm{HT}$ for $24 \mathrm{~h}$ and cultured with $100 \mathrm{ng} / \mathrm{ml}$ TNF alone or TNF plus $500 \mathrm{nM}$ smac-mimetic for $48 \mathrm{~h}$. Cells were resuspended using trypsin, re-plated, and after 5 days stained with crystal violet to assess clonogenicity

in which both RIPK3 and MLKL were induced increased the amount of cell death (Figure 6b). Therefore, in these cells ligated TNFR1 was able to activate RIPK3 and MLKL in the absence of FADD. Furthermore, we could show upon induction of RIPK3, MLKL, and TNF treatment, there was no caspase 8 cleavage in the Fadd $^{-1-}$ MEFs as compared to WT MEFs with RIPK3 overexpression and TNF treatment (Figure 6c). Therefore, in order for RIPK3 to induce activation and cleavage of caspase 8 , it requires FADD.

\section{Discussion}

We set out to determine the requirements for particular signaling components involved in cell death in response to TNF. To do so, we used MEFs derived from gene-deleted mice. Consistent with many earlier reports, addition of TNF to WT MEFs did not cause them to die, ${ }^{29,34}$ but when the cells had been treated with a smac-mimetic compound that depletes cells of cIAP1, addition of TNF did cause cell death. 
As TNF plus smac-mimetic did not kill Ripk1-1- or caspase $8^{-1-}$ MEFs, which were able to divide and form colonies, clAP1 in WT MEFs must prevent activation of a TNF-induced death pathway that requires both RIPK1 and caspase 8.

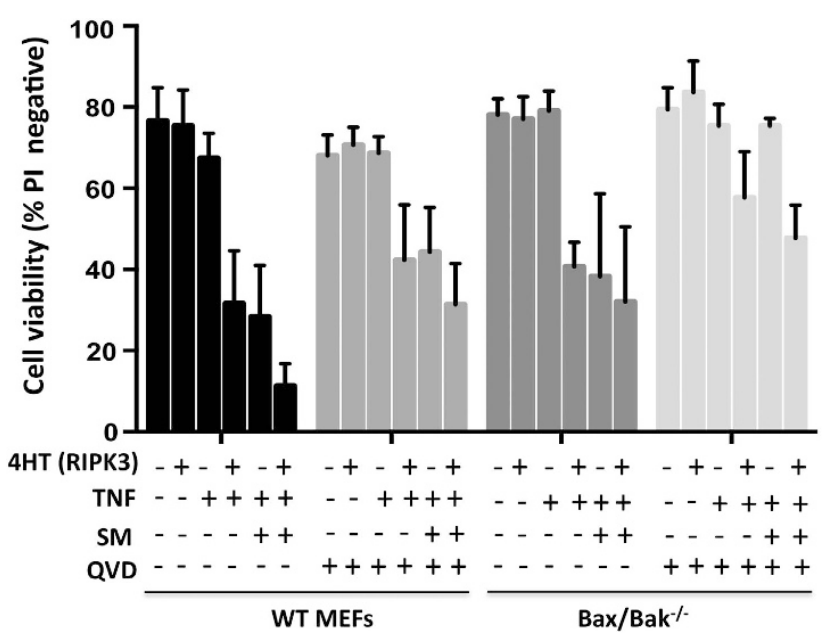

Figure 4 Death induced by TNF and activated RIPK3 is independent of proapoptotic Bcl-2 family proteins Bax and Bak. WT and $\mathrm{Bax}^{-1-} / \mathrm{Bak}^{-1-} \mathrm{MEFs}$ bearing the inducible FLAG-RIPK3 vector were induced with $10 \mathrm{nM} 4 \mathrm{HT}$ for $24 \mathrm{~h}$. Where indicated, cells were pretreated with $10 \mu \mathrm{M}$ Q-VD-OPh for $1 \mathrm{~h}$ and subsequently treated with $100 \mathrm{ng} / \mathrm{ml}$ TNF or TNF plus $500 \mathrm{nM}$ smac-mimetic (SM) for $24 \mathrm{~h}$. Cells were stained with PI and cell viability determined by flow cytometry. Columns show mean \pm S.E.M., where $n=3$ independently performed experiments
Although the MEF lines expressed endogenous RIPK3, to determine if overexpression of RIPK3 alone was sufficient to induce necroptosis, we expressed additional RIPK3 from an inducible lentiviral vector. Overexpression of RIPK3 alone was not sufficient to cause death of MEFs, but unlike WT cells, cells with increased RIPK3 died upon addition of TNF. We found that when RIPK3 levels were increased, TNF triggered activation of caspase 8, whether RIPK1 was present or not. These results show that when ligated, TNFR1 can activate RIPK3, and thereby cause activation of caspases 8 and 3, cleavage of PARP, and cell death, even when RIPK1 was absent.

These findings question prior assumptions that because it bears a DD, RIPK1 was essential for TNF to induce activation of RIPK3, or was essential for TNF to activate caspase 8. Although we do not know precisely how TNFR1 activates RIPK3 in the absence of RIPK1, TRADD appears to be necessary, but to date there have been no reports showing that TRADD can bind to RIPK3. TRAF2 might also be responsible for recruiting RIPK3 into death-inducing complexes, as TRAF2 is rapidly recruited to complex I upon ligation of TNFR1, and RIPK3 has been reported to co-immunopreciptitate with TRAF2. ${ }^{35}$

We initially wondered if FADD might be responsible for recruiting RIPK3 to death-inducing complexes in the Ripk $1^{-1}$ MEFs, firstly because some preliminary experiments suggested that Fadd $^{-1-}$ MEFs did not die when RIPK3 was elevated and cells were treated with TNF, and secondly because it has been reported that FADD can interact directly with RIPK3. ${ }^{14,23}$ However, the Fadd ${ }^{-1-}$ line had relatively low
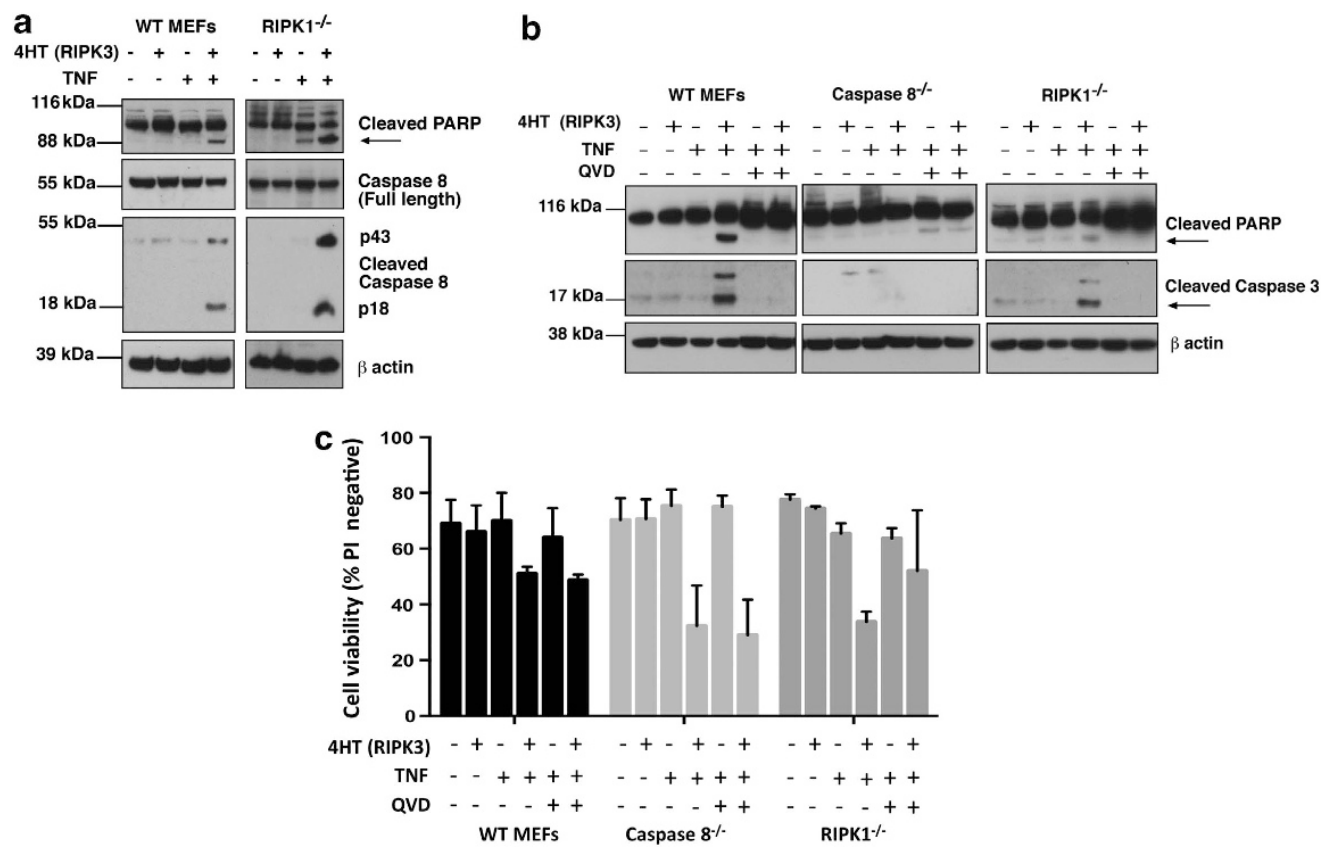

Figure 5 TNF treatment of cells overexpressing RIPK3 activates caspases, but this is not necessary for cell death. (a) WT and Ripk $1^{-1-}$ MEFs were induced with $10 \mathrm{nM}$ $4 \mathrm{HT}$ for $24 \mathrm{~h}$ and treated with $100 \mathrm{ng} / \mathrm{ml}$ TNF. After $4 \mathrm{~h}$, cells were harvested, lysed, and analyzed by western blotting, and probed for poly (ADP-ribose) polymerase (PARP) and both full-length and cleaved caspase 8 . $\beta$-Actin was used as a loading control. (b, c) WT (caspase $8^{\text {lox/lox}}$ ), caspase $8^{-1-}$, and Ripk $1^{-1-}$ MEFs in duplicate wells were induced with $10 \mathrm{nM} 4 \mathrm{HT}$ for $24 \mathrm{~h}$ and treated with $100 \mathrm{ng} / \mathrm{ml} \mathrm{TNF}$. (b) After $4 \mathrm{~h}$, cells from one set of wells were harvested, lysed, and analyzed by western blot probed for PARP and cleaved caspase 3. (c) After $4 \mathrm{~h}$ in culture, the cells in the second set of wells were re-suspended, stained with $100 \mathrm{ng} / \mathrm{ml} \mathrm{PI}$, and cell viability determined by flow cytometry. Columns show mean \pm S.E.M., where $n=3$ independently performed experiments 


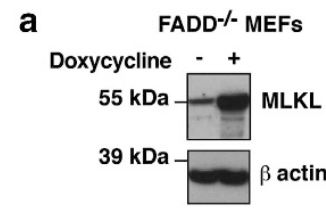

b
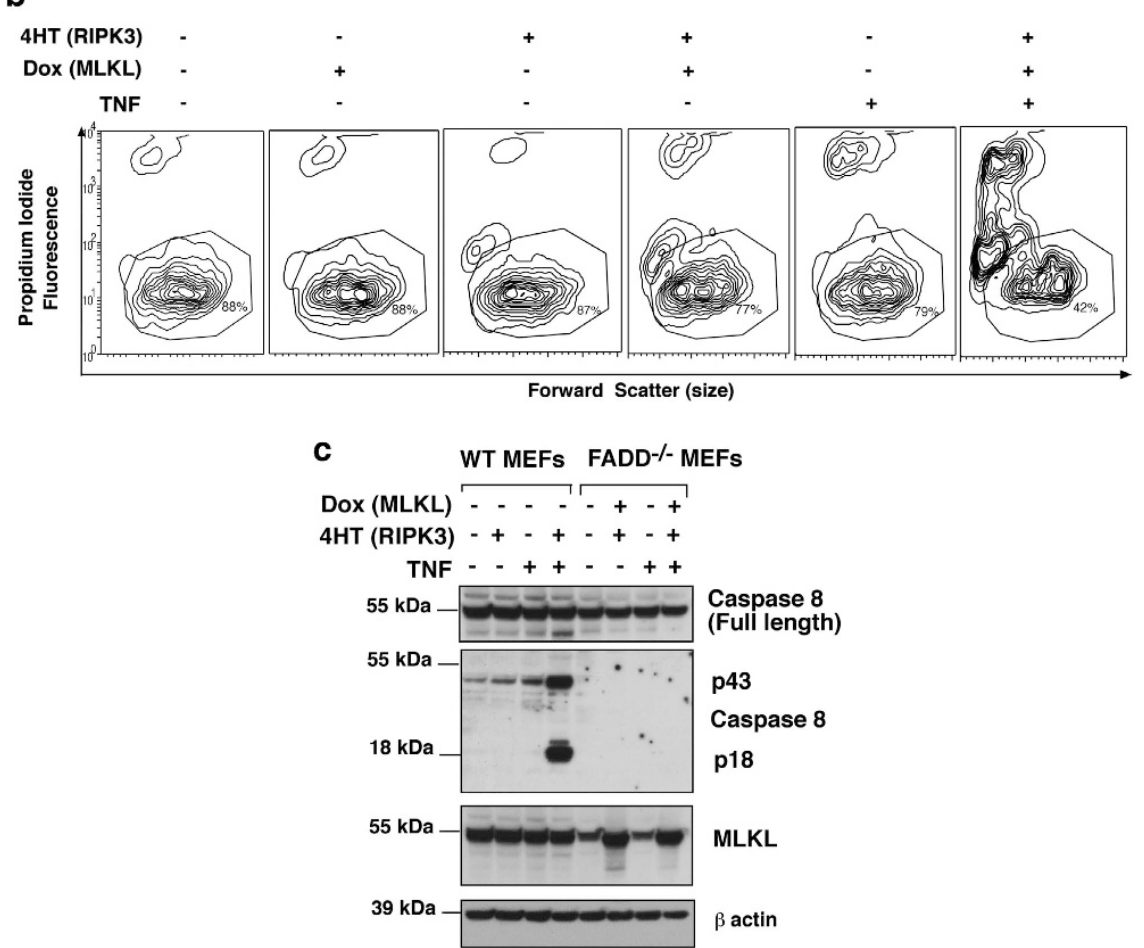

Figure 6 Elevated levels of MLKL in Fadd ${ }^{-1}$ MEFs allows cell death by TNF-induced activation of RIPK3. (a) Fadd ${ }^{-1-}$ MEFs were infected with a doxycycline (dox)-inducible MLKL expression vector. Cells were treated with $1 \mu \mathrm{g} / \mathrm{ml}$ of dox for $24 \mathrm{~h}$. Cell lysates were harvested and probed for MLKL as indicated and $\beta$-actin was used as a loading control. (b) Fadd ${ }^{-1}$ MEFs bearing vectors for dox-inducible MLKL and 4HT-inducible RIPK3 were treated with $1 \mu \mathrm{g} / \mathrm{ml}$ dox, $10 \mathrm{nM} 4 \mathrm{HT}$, and $100 \mathrm{ng} / \mathrm{ml}$ TNF for $24 \mathrm{~h}$. Cells were re-suspended using trypsin, stained with $100 \mathrm{ng} / \mathrm{ml} \mathrm{PI}$, and analyzed by flow cytometry. (c) WT MEFs bearing a $4 \mathrm{HT}$-inducible RIPK3 vector and Fadd ${ }^{-1-}$ MEFs bearing vectors for $4 \mathrm{HT}$-inducible RIPK3 and dox-inducible MLKL were treated with $1 \mu \mathrm{g} / \mathrm{ml}$ dox and $10 \mathrm{nM} 4 \mathrm{HT}$ for $24 \mathrm{~h}$ and treated with $100 \mathrm{ng} / \mathrm{ml}$ TNF for $4 \mathrm{~h}$. Cell lysates were harvested and probed for caspase 8 (full-length), cleaved caspase 8 and MLKL as indicated, and $\beta$-actin was used as a loading control

levels of MLKL, and when MLKL levels were elevated, TNF was able to kill the Fadd $^{-1-}$ cells. These results suggest that FADD might be needed for activation of caspase 8 , but is not required for TNFR1 to activate RIPK3 and MLKL to cause necroptosis.

The necroptotic cell death pathway triggered by TNF in cells with elevated RIPK3 did not require the presence of FADD, RIPK1, caspase 8, or Bax/Bak. The ability of induced MLKL to restore the sensitivity of the Fadd ${ }^{-1-}$ cells is consistent with MLKL being activated by RIPK3 and having an important role in necroptosis. ${ }^{22}$ Although how MLKL kills has not yet been determined with certainty, our observations are consistent with reports that it causes cell death by a caspase- and Bax/ Bak-independent mechanism. ${ }^{17,22}$

Our results are consistent with the model shown in Figure 7. WT MEFs remain viable when TNF is added, because cIAP1, TRAF2, and RIPK1 allow activation of canonical NF- $\kappa \mathrm{B}$ pathways resulting in the expression of cell death inhibitors such as FLIP, clAP2, and $A 20^{28,36,37}$ (panel 1). When smac-mimetic is added, it causes autoubiquitylation and degradation of IAPS, so that cells treated with TNF plus smac-mimetic die by a caspase 8- and RIPK1-dependent mechanism (panel 2).

When levels of RIPK3 are elevated (panel 3), ligation of TNFR1 signals via TRADD to activate RIPK3, and TNF can trigger RIPK3 activation even when RIPK1 is absent. Once activated, RIPK3 can in turn activate two distinct cell death pathways, one involving caspase 8 , and another that involves MLKL (panel 3). In Fadd ${ }^{-1-}$ MEFs, ligation of TNFR1 can activate RIPK3 when its levels are elevated, and it can activate the necroptotic pathway but not the caspase 8-dependent cell death pathway (panel 4).

These findings do not exclude the possibility that RIPK1 can activate RIPK3; indeed, this is likely to be what occurs when necrostatin is able to inhibit cell death. ${ }^{11,19}$ These findings are also consistent with a model in which RIPK1 acts in parallel with RIPK3 such that in the presence of RIPK1, lower levels of RIPK3 are needed for necroptosis. However, our results provide strong evidence that RIPK1 does not have an obligatory role in necroptosis signaling, challenging models 


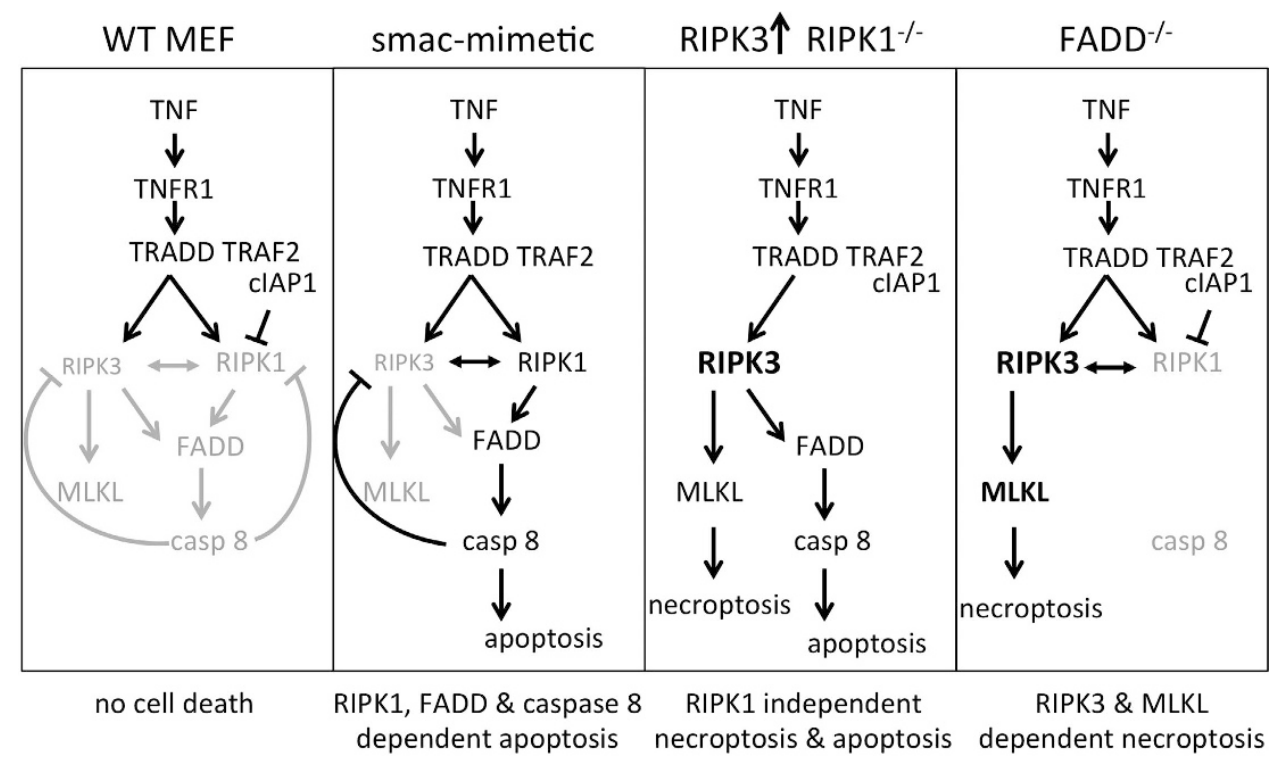

Figure 7 Model for activation of caspase-dependent and -independent cell death mechanisms by TNF. Under normal conditions, TNF treatment of WT MEFs does not induce cell death. When IAPs are depleted with smac-mimetic, TNF can activate caspase 8 by a pathway requiring RIPK1 to cause cell death that can be blocked by the caspase inhibitor Q-VD-OPh. When levels of RIPK3 are elevated, TNF not only activates caspase 8 but also activates a caspase-independent death mechanism, but it does not require RIPK1 for either

which suggested that RIPK1 has an essential role upstream of RIPK3 and is required for its activation.

\section{Materials and Methods}

Genetically modified cell lines and mice. Production of MEF lines has been described previously in detail. ${ }^{7}$ Briefly, primary MEFs were generated from E15.5 embryos and then infected with SV40 large T antigen-expressing lentivirus to generate immortal cell lines. Ripk $1^{-1-}$ mice were provided by Michelle Kelliher (University of Massachusetts Medical School, Worcester, MA, USA) and Tnfr1 ${ }^{-1-}$ and Tnfr2 $^{-1-}$ mice were a kind gift from Heinrich Korner (University of Tasmania, Sandy Bay, TAS, Australia). Fadd ${ }^{-1-}$ MEFs were provided by Francis Chan (University of Massachusetts Medical School), Tradd ${ }^{-1-}$ MEFs were obtained from Henning Walczak (Imperial College, London, UK) and Manolis Pasparakis (University of Cologne, Cologne, Germany), and Bax/Bak ${ }^{-1-}$ MEFs were provided by David Huang (Walter and Eliza Hall Institute, Parkville, VIC Australia). Caspase 8 conditional knockin mice were provided by Stephen Hedrick (University of California San Diego, San Diego, CA, USA).

Generation of caspase $\mathbf{8}^{-/-}$MEFs. Caspase 8 gene-deleted MEFs were generated from E8.5 caspase 8 LoxP/LoxP embryos (Dr. Steve Hedrick, University of California San Diego). Primary MEFs were immortalized by infection with SV40 largeT antigen-expressing lentivirus as described previously. ${ }^{7}$ To delete caspase 8 , the transformed MEFs were infected with a Cre-recombinaseexpressing lentivirus (pFU CreR SV40 Hygro) and deletion was confirmed by western blot.

Cell culture, transfections, constructs and lentiviral infections. Cell lines were maintained at $37^{\circ} \mathrm{C}, 10 \% \mathrm{CO}_{2}$ in DMEM supplemented with $10 \%$ (v/v) fetal bovine serum (Gibco, Melbourne, VIC, Australia), $50 \mu \mathrm{g} / \mathrm{ml}$ penicillin G, $50 \mathrm{U} / \mathrm{ml}$ streptomycin, and $2 \mathrm{mM} \mathrm{L-glutamine.} \mathrm{Medium} \mathrm{for} \mathrm{Bax/Bak}{ }^{-1-}$ MEFs was also supplemented with $270 \mu \mathrm{M}$ L-asparagine and $50 \mu \mathrm{M}$ 2-mercaptoethanol. Crerecombinase and SV40 large T antigen were cloned into the lentiviral vector pFU as described previously. ${ }^{7}$ Mouse FLAG-RIPK3 was cloned into the $4 \mathrm{HT}$-inducible lentiviral vector $\mathrm{pF} 5 \times \mathrm{UAS}^{7,38}$ Lentiviruses were generated by transfecting subconfluent $10 \mathrm{~cm}$ plates of $293 \mathrm{~T}$ cells with vector plasmids together with the packaging constructs pCMV- $\Delta R 8$ and pVSV-G using Effectene (Qiagen, Chadstone, VIC, Australia) as described previously. ${ }^{38}$ After $48 \mathrm{~h}$, viral supernatants were collected, filtered, supplemented with $4 \mu \mathrm{g} / \mathrm{ml}$ polybrene, and added to the target MEFs. Stably infected cells were selected in the presence of $5 \mu \mathrm{g} / \mathrm{ml}$ puromycin (puro) and $300 \mu \mathrm{g} / \mathrm{ml}$ hygromycin B. Expression of pF $5 \times$ UASinducible constructs was induced with $10 \mathrm{nM} 4 \mathrm{HT}$, unless otherwise indicated. The doxycycline-inducible vector $\mathrm{pF}$ TRE3G PGK puro is a derivative of the pRetroX retroviral vector (Clontech, Mountain View, CA, USA) possessing a Tet-On 3G transactivator (Tet3G), a puro resistance gene expressed under the control of a PGK promoter, and a tetracycline-responsive element. ${ }^{39}$ The cDNA-encoding mouse MLKL (residues 1-464; UniProt sequence Q9D2Y4-2) was PCR amplified using the following primers: forward, $5^{\prime}$-CGCGGATCCAAGCCACCATGGCGCGC CAGGAC-3'; reverse, 5'-CGCGGATCCTTACACCTTCTTGTCCGTGGATTC-3'. The BamHI-digested PCR product was ligated into the BamHI site of the $\mathrm{pF}$ TRE3G PGK puro vector and was verified by sequencing (Micromon DNA Sequencing Facility, Clayton, VIC, Australia).

Antibodies and chemicals. Primary antibodies used for western blot analysis were anti-FLAG (F-3165; Sigma, Croydon, VIC, Australia), anti- $\beta$-actin (A1978; Sigma), anti-RIPK1 (610458; BD Transduction Laboratories, North Ryde, NSW, Australia), anti-RIPK3 (551042; Pharmingen, North Ryde, NSW, Australia), anticaspase 8 (Lorraine O'Reilly, The Walter and Eliza Hall Institute, Melbourne, VIC, Australia), anticleaved caspase 8 (Asp 387) (8592; Cell Signaling Technology, Arundel, QLD, Australia), anticleaved caspase 3 (Asp 175) (9661; Cell Signaling Technology), anti-PARP (9542; Cell Signaling Technology, Arundel, Queensland Australia), and anti-MLKL (Jian-Guo Zhang, The Walter and Eliza Hall Institute). 4HT and doxycycline were purchased from Sigma, Fc-TNF (in-house) and Q-VD-OPh (OPH001) from SM Biochemicals, (Anaheim, CA, USA) and smac-mimetic was obtained from TetraLogic Pharmaceuticals (Malvern, PA, USA).

Cell death assays. Cells were seeded at approximately $40 \%$ confluency onto 12-well tissue culture plates and were allowed to settle for $16-20 \mathrm{~h}$. Where indicated, $500 \mathrm{nM}$ smac-mimetic (Compound A; TetraLogic Pharmaceuticals), $100 \mathrm{ng} / \mathrm{ml}$ human Fc-TNF, $10 \mathrm{nM} 4 \mathrm{HT}$, and/or $10 \mu \mathrm{M} \mathrm{Q}$-VD-OPh were added to cells for $24 \mathrm{~h}$ and cell death measured by uptake of propidium iodide (PI) using a FACScalibur flow cytometer (BD Biosciences, North Ryde, NSW, Australia). In all, 10000 events per sample were collected, and the percentage of live cells (\% PI-negative cells) was quantified using WEASEL software (version 2.2.2; WEHI).

Clonogenic survival assay. WT, caspase $8^{-1-}, R i p k 1^{-1-}$, and Bax $B a k^{-1-}$ MEFs were plated at equal densities on six-well plates, and cultured with or without TNF and smac-mimetic for $24 \mathrm{~h}$. After treatment, cells were treated with trypsin, re-suspended, washed, and re-plated. Cells were then grown 
for 5 days and fixed with glutaraldehyde, and colonies stained with $0.1 \%(\mathrm{w} / \mathrm{v})$ crystal violet.

Western blotting. Lysates were prepared in DISC lysis buffer $(20 \mathrm{mM}$ Tris- $\mathrm{HCl}$ (pH 7.4), $150 \mathrm{mM} \mathrm{NaCl}, 10 \%$ glycerol, 10\% Triton X-100), supplemented with protease inhibitor cocktail (Roche, Dee Why,NSW Australia). Protein samples were separated on 4-12\% polyacrylamide gels (Invitrogen, Mulgrave, VIC, Australia), and transferred to Hybond C nitrocellulose membrane (GE, Rydalmere, NSW, Australia) for incubation with specified antibodies. All membrane blocking steps and antibody dilutions were performed using $5 \%(\mathrm{v} / \mathrm{v})$ skim milk in PBS containing $0.1 \%(\mathrm{v} / \mathrm{v})$ Tween-20 phosphate-buffered saline (PBST), and washing steps performed with PBST. Western blots were visualized by enhanced chemiluminescence (GE).

\section{Conflict of Interest}

The authors declare no conflict of interest.

Acknowledgements. This work was funded by NHMRC grants and fellowships 433063, 461221, 541901, 575512, 637342, 1003435, and ARC fellowship FT100100100, and was made possible through Victorian State Government Operational Infrastructure Support and Australian Government NHMRC IRIISS. We thank Stephen Hedrick, Michelle Kelliher, and Heinrich Korner for caspase 8, TRADD, FADD, and RIPK1 gene-deleted cell lines and mice, and Mark McKinlay (TetraLogic Pharmaceuticals) for smac-mimetic compound. We would also like to acknowledge Ian Gentle for helping construct the 4HT-inducible FLAG RIPK3 construct.

1. Baud V, Karin M. Signal transduction by tumor necrosis factor and its relatives. Trends Cell Biol 2001; 11: 372-377.

2. Laster SM, Wood JG, Gooding LR. Tumor necrosis factor can induce both apoptic and necrotic forms of cell lysis. J Immunol 1988; 141: 2629-2634.

3. Beg AA, Baltimore D. An essential role for Nf-Kappa-B in preventing Tnf-alpha-induced cell death. Science 1996; 274: 782-784.

4. Chinnaiyan AM, Tepper CG, Seldin MF, Orourke K, Kischkel FC, Hellbardt S et al. Fadd/ mort1 is a common mediator of cd95 (fas/apo-1) and tumor necrosis factor receptorinduced apoptosis. J Biol Chem 1996; 271: 4961-4965.

5. Varfolomeev EE, Boldin MP, Goncharov TM, Wallach D. A potential mechanism of cross-talk between the p55 tumor necrosis factor receptor and fas/apo1 - proteins binding to the death domains of the two receptors also bind to each other. J Exp Med 1996; 183: $1271-1275$.

6. Muzio M, Stockwell BR, Stennicke HR, Salvesen GS, Dixit VM. An induced proximity model for caspase-8 activation. J Biol Chem 1998; 273: 2926-2930.

7. Vince JE, Wong WW, Khan N, Feltham R, Chau D, Ahmed AU et al. IAP antagonists target ClAP1 to induce TNFalpha-dependent apoptosis. Cell 2007; 131: 682-693.

8. Zhou Q, Snipas S, Orth K, Muzio M, Dixit VM, Salvesen GS. Target protease specificity of the viral serpin crma - analysis of five caspases. J Biol Chem 1997; 272: 7797-7800.

9. Caserta TM, Smith AN, Gultice AD, Reedy MA, Brown TL. Q-VD-OPh a broad spectrum caspase inhibitor with potent antiapoptotic properties. Apoptosis 2003; 8: 345-352.

10. Kunstle G, Leist M, Uhlig S, Revesz L, Feifel R, Mackenzie A et al. Ice-protease inhibitors block murine liver injury and apoptosis caused by cd95 or by tnf-alpha. Immunol Lett 1997; 55: 5-10.

11. Degterev A, Huang Z, Boyce M, Li Y, Jagtap P, Mizushima $N$ et al. Chemical inhibitor of nonapoptotic cell death with therapeutic potential for ischemic brain in jury. Nat Chem Biol 2005; 1: 112-119.

12. Vercammen D, Beyaert R, Denecker G, Goossens V, Van Loo G, Declercq W et al. Inhibition of caspases increases the sensitivity of L929 cells to necrosis mediated by tumor necrosis factor. J Exp Med 1998; 187: 1477-1485.

13. Declercq W, Vanden Berghe T, Vandenabeele P. RIP kinases at the crossroads of cell death and survival. Cell 2009; 138: 229-232.

14. Cho YS, Challa S, Moquin D, Genga R, Ray TD, Guildford M et al. Phosphorylation-driven assembly of the RIP1-RIP3 complex regulates programmed necrosis and virus-induced inflammation. Cell 2009; 137: 1112-1123.

15. Feoktistova M, Geserick P, Kellert B, Dimitrova DP, Langlais C, Hupe M et al. clAPs block ripoptosome formation, a RIP1/caspase-8 containing intracellular cell death complex differentially regulated by cFLIP isoforms. Mol Cell 2011; 43: 449-463.

16. Tenev T, Bianchi K, Darding M, Broemer M, Langlais C, Wallberg F et al. The ripoptosome, a signaling platform that assembles in response to genotoxic stress and loss of IAPs. Mol Cell 2011; 43: 432-448.

17. Wang Z, Jiang H, Chen S, Du F, Wang X. The mitochondrial phosphatase PGAM5 functions at the convergence point of multiple necrotic death pathways. Cell 2012; 148: 228-243.

18. Zhang DW, Shao J, Lin J, Zhang N, Lu BJ, Lin SC et al. RIP3, an energy metabolism regulator that switches TNF-induced cell death from apoptosis to necrosis. Science 2009; 325: 332-336.

19. Degterev A, Hitomi J, Germscheid M, Ch'en IL, Korkina O, Teng X et al. Identification of RIP1 kinase as a specific cellular target of necrostatins. Nat Chem Biol 2008; 4: 313-321.

20. Sun X, Lee J, Navas T, Baldwin DT, Stewart TA, Dixit VM. RIP3 a novel apoptosis-inducing kinase. J Biol Chem 1999; 274: 16871-16875.

21. Micheau O, Tschopp J. Induction of TNF receptor I-mediated apoptosis via two sequential signaling complexes. Cell 2003; 114: 181-190.

22. Sun L, Wang H, Wang Z, He S, Chen S, Liao D et al. Mixed lineage kinase domain-like protein mediates necrosis signaling downstream of RIP3 kinase. Cell 2012; 148: 213-227.

23. Vanlangenakker N, Vanden Berghe T, Bogaert P, Laukens B, Zobel K, Deshayes K et al. CIAP1 and TAK1 protect cells from TNF-induced necrosis by preventing RIP1/RIP3dependent reactive oxygen species production. Cell Death Differ 2011; 18: 656-665.

24. He S, Wang L, Miao L, Wang T, Du F, Zhao L et al. Receptor interacting protein kinase-3 determines cellular necrotic response to TNF-alpha. Cell 2009; 137: 1100-1111.

25. Kaiser WJ, Offermann MK. Apoptosis induced by the toll-like receptor adaptor TRIF is dependent on its receptor interacting protein homotypic interaction motif. J Immunol 2005; 174: 4942-4952.

26. Li J, McQuade T, Siemer AB, Napetschnig J, Moriwaki K, Hsiao YS et al. The RIP1/RIP3 necrosome forms a functional amyloid signaling complex required for programmed necrosis. Cell 2012; 150: 339-350.

27. Vandenabeele P, Declercq W, Van Herreweghe F, Vanden Berghe T. The role of the kinases RIP1 and RIP3 in TNF-induced necrosis. Sci Signal 2010; 3: 4.

28. Moujalled DM, Cook WD, Lluis JM, Khan NR, Ahmed AU, Callus BA et al. In mouse embryonic fibroblasts, neither caspase-8 nor cellular FLICE-inhibitory protein (FLIP) is necessary for TNF to activate NF-kappaB, but caspase-8 is required for TNF to cause cell death, and induction of FLIP by NF-kappaB is required to prevent it. Cell Death Differ 2011; 5: 808-815.

29. Wong WW, Gentle IE, Nachbur U, Anderton H, Vaux DL, Silke J. RIPK1 is not essential for TNFR1-induced activation of NF-kappaB. Cell Death Differ 2009; 3: 482-487.

30. Abhari BA, Cristofanon S, Kappler R, von Schweinitz D, Humphreys R, Fulda S. RIP1 is required for IAP inhibitor-mediated sensitization for TRAIL-induced apoptosis via a RIP1/ FADD/caspase-8 cell death complex. Oncogene 2012; e-pub ahead of print, 13 August 2012; doi:10.1038/onc.2012.337.

31. Upton JW, Kaiser WJ, Mocarski ES. DAI/ZBP1/DLM-1 complexes with RIP3 to mediate virus-induced programmed necrosis that is targeted by murine cytomegalovirus vIRA. Cell Host Microbe 2012; 11: 290-297.

32. Irrinki KM, Mallilankaraman K, Thapa RJ, Chandramoorthy HC, Smith FJ, Jog NR et al. Requirement of FADD, NEMO, and BAX/BAK for aberrant mitochondrial function in tumor necrosis factor alpha-induced necrosis. Mol Cell Biol 2011; 31: 3745-3758.

33. Zhao J, Jitkaew S, Cai Z, Choksi S, Li Q, Luo J et al. Mixed lineage kinase domain-like is a key receptor interacting protein 3 downstream component of TNF-induced necrosis. Proc Natl Acad Sci USA 2012; 109: 5322-5327.

34. Gentle IE, Wong WW, Evans JM, Bankovacki A, Cook WD, Khan NR et al. In TNFstimulated cells, RIPK1 promotes cell survival by stabilizing TRAF2 and cIAP1, which limits induction of non-canonical NF-kappaB and activation of caspase-8. J Biol Chem 2011; 286: 13282-13291.

35. Yu PW, Huang BC, Shen M, Quast J, Chan E, Xu X et al. Identification of RIP3, a RIP-like kinase that activates apoptosis and NFkappaB. Curr Biol 1999; 9: 539-542.

36. Chu ZL, Mckinsey TA, Liu L, Gentry JJ, Malim MH, Ballard DW. Suppression of tumor necrosis factor-induced cell death by inhibitor of apoptosis c-iap2 is under nf-kappa-b control. Proc Natl Acad Sci USA 1997; 94: 10057-10062.

37. He KL, Ting AT. A20 inhibits tumor necrosis factor (TNF) alpha-induced apoptosis by disrupting recruitment of TRADD and RIP to the TNF receptor I complex in Jurkat T cells. Mol Cell Biol 2002; 22: 6034-6045

38. Callus BA, Moujallad DM, Silke J, Gerl R, Jabbour AM, Ekert PG et al. Triggering of apoptosis by Puma is determined by the threshold set by prosurvival Bcl-2 family proteins. J Mol Biol 2008; 384: 313-323.

39. Yamamoto M, Okuyama M, Ma JS, Kimura T, Kamiyama N, Saiga $\mathrm{H}$ et al. A cluster of interferon-gamma-inducible p65 GTPases plays a critical role in host defense against Toxoplasma gondii. Immunity 2012; 37: 302-313.

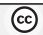

SOMERIAHTS

published by Nature Publishing Group. This work is licensed under the Creative Commons Attribution-NonCommercial-No Derivative Works 3.0 Unported License. To view a copy of this license, visit http://creativecommons.org/licenses/by-nc-nd/3.0/ 